application of AT1R antagonism as a therapy for patients with LDS. There is both retrospective and evolving prospective evidence that AT1R antagonism may be beneficial to patients with Marfan syndrome (6, $18,19)$. Together, the findings in patients with Marfan syndrome and the results from the Gallo et al. study (12) indicate that patients with LDS may potentially benefit from AT1R antagonism. Losartan has been the ARB of choice in most ongoing trials; however, the use of an ARB with a more favorable pharmacokinetic profile and longer half-life may enhance the protective effects against TAAs. The availability of the LDS mouse described by Gallo et al. (12) provides a model to determine the relative efficacies of this class of drugs before application to humans.

\section{Acknowledgments}

Frank Davis is supported by a Sarnoff Cardiovascular Foundation Fellowship. Research work is supported by funding from the NIH (HL062846 and HL107319).

Address correspondence to: Alan Daugherty, Saha Cardiovascular Research Center, Biomedical Biological Sciences Research Building, B243, University of Kentucky,
Lexington, Kentucky 40536-0509, USA.

Phone: 859.323.3512; Fax: 859.257.3235;

E-mail: Alan.Daugherty@uky.edu.

1. Lindsay ME, Dietz HC. Lessons on the pathogenesis of aneurysm from heritable conditions. Nature. 2011;473(7347):308-316.

2. Milewicz DM, Dietz HC, Miller DC. Treatment of aortic disease in patients with Marfan syndrome. Circulation. 2005;111(11):e150-e157.

3. Habashi JP, et al. Losartan, an AT1 antagonist, prevents aortic aneurysm in a mouse model of Marfan syndrome. Science. 2006;312(5770):117-121.

4. Habashi JP, et al. Angiotensin II type 2 receptor signaling attenuates aortic aneurysm in mice through ERK antagonism. Science. 2011;332(6027):361-365.

5 . Holm TM, et al. Noncanonical TGF- $\beta$ signaling contributes to aortic aneurysm progression in Marfan syndrome mice. Science. 2011;332(6027):358-361.

6. Moltzer E, Essers J, van Esch JH, Roos-Hesselink JW, Danser AH. The role of the renin-angiotensin system in thoracic aortic aneurysms: clinical implications. Pharmacol Ther. 2011;131(1):50-60.

7. Chen X, Lu H, Rateri DL, Cassis LA, Daugherty A. Conundrum of angiotensin II and TGF- $\beta$ interactions in aortic aneurysms. Curr Opin Pharmacol. 2013;13(2):180-185.

8. Loeys BL, et al. Aneurysm syndromes caused by mutations in the TGF- $\beta$ receptor. NEnglJ Med. 2006; 355(8):788-798.

9. Kalra VB, Gilbert JW, Malhotra A. Loeys-Dietz syndrome: cardiovascular, neuroradiological and musculoskeletal imaging findings. Pediatr Radiol. 2011;41(12):1495-1504

10. Gillis E, Van Laer L, Loeys BL. Genetics of thoracic aortic aneurysm: at the crossroad of transforming growth factor- $\beta$ signaling and vascular smooth muscle cell contractility. Circ Res. 2013;113(3):327-340.
11. Lindsay ME, et al. Loss-of-function mutations in TGFB2 cause a syndromic presentation of thoracic aortic aneurysm. Nat Genet. 2012;44(8):922-927.

12. Gallo EM, et al. Angiotensin II-dependent TGF- $\beta$ signaling contributes to Loeys-Dietz syndrome vascular pathogenesis. J Clin Invest. 2014; 124(1):448-460.

13. Rodríguez-Vita J, Sánchez-López E, Esteban V, Rupérez M, Egido J, Ruiz-Ortega M. Angiotensin II activates the Smad pathway in vascular smooth muscle cells by a transforming growth factor- $\beta$-independent mechanism. Circulation. 2005;111(19):2509-2517.

14. Sadoshima J. Novel AT(1) receptor-independent functions of losartan. Circ Res. 2002;90(7):754-756.

15. Subramanian V, Golledge J, Heywood EB, Bruemmer D, Daugherty A. Regulation of peroxisome proliferator-activated receptor-gamma by angiotensin II via transforming growth factor- $\beta 1$-activated p38 mitogen-activated protein kinase in aortic smooth muscle cells. Arterioscler Thromb Vasc Biol. 2012; 32(2):397-405.

16. Zhang XH, Zheng B, Gu C, Fu JR, Wen JK. TGF- $\beta 1$ downregulates AT1 receptor expression via PKC- $\delta$-mediated Sp1 dissociation from KLF4 and Smad-mediated PPAR- $\gamma$ association with KLF4. Arterioscler Thromb Vasc Biol. 2012;32(4):1015-1023.

17. Kuang SQ, et al. Aortic remodeling after transverse aortic constriction in mice is attenuated with AT1 receptor blockade. Arterioscler Thromb Vasc Biol. 2013; 33(9):2172-2179.

18. Brooke BS, Habashi JP, Judge DP, Patel N, Loeys B. Angiotensin II blockade and aortic-root dilation in Marfan's syndrome. N Engl J Med. 2008; $358(26): 2787-2795$.

19. Groenink M, et al. Losartan reduces aortic dilatation rate in adults with Marfan syndrome: a randomized controlled trial [published online ahead of print September 2, 2013]. Eur Heart J. doi:10.1093/eurheartj/eht334.

\title{
Toward postnatal reversal of ocular congenital malformations
}

\author{
José-Alain Sahel1,2,3,4,5,6,7 and Katia Marazova1,2,3
}

1INSERM, U968, Paris, France. 2Université Pierre et Marie Curie-Paris 6, UM80, Institut de la Vision, Paris, France. ${ }^{3}$ CNRS, UMR 7210, Paris, France. ${ }^{4}$ Centre Hospitalier National d'Ophtalmologie des Quinze-Vingts, INSERM-DHOS CIC 503, Paris, France. ${ }^{5}$ Fondation Ophtalmologique Adolphe de Rothschild, Paris, France. 6 Institute of Ophthalmology, University College of London, London, United Kingdom. ${ }^{7}$ French Academy of Sciences, Institut de France, Paris, France.

\begin{abstract}
Aniridia is a panocular disorder that severely affects vision in early life. Most cases are caused by dominantly inherited mutations or deletions of the PAX6 gene, which encodes a transcription factor that is essential for the development of the eye and the central nervous system. In this issue of the JCI, Gregory-Evans and colleagues demonstrate that early postnatal topical administration of an ataluren-based formulation reverses congenital malformations in the postnatal mouse eye, providing evidence that manipulation of PAX6 after birth may lead to corrective tissue remodeling. These findings offer hope that ataluren administration could be a therapeutic paradigm applicable to some major congenital eye defects.
\end{abstract}

Conflict of interest: José-Alain Sahel is a founder of and consultant for GenSight and Pixium Vision and a consultant for Sanofi and Gene Signal.

Citation for this article: $J$ Clin Invest. 2014; 124(1):81-84. doi:10.1172/JCI73560.
Mutations that inactivate gene function by promoting premature translational termination cause a large number of human diseases. It is thought that at least one-third of all genetic diseases and many types of can- cer are the result of such mutations $(1,2)$. These mutations are referred to as nonsense mutations, premature stop mutations, or premature termination codons (PTCs). Given that PTCs often result in a complete loss of protein function, the associated diseases usually manifest as severe phenotypes. Examples of PTC-associated diseases include CF, Duchenne muscular dystrophy (DMD), and aniridia, among others.

\section{Aniridia, a panocular disorder}

Aniridia is a rare eye disease with an estimated prevalence of approximately 1 in 40,000 to 1 in 100,000 individuals. It is present at birth and characterized by a total 
or partial absence of iris tissue and foveal hypoplasia that results in reduced visual acuity, with eyesight in the range of 20/100 to $20 / 200$, and nystagmus (reviewed in ref. 3). There are multiple ocular abnormalities associated with aniridia, although these vary within and between families. The associated ocular defects usually manifest later in life and may include cataract, glaucoma, corneal opacification and vascularization, lens subluxation, strabismus, and optic nerve coloboma and hypoplasia. Patients with aniridia may have other sensory deficits, including reduced olfaction, hearing difficulties, and, rarely, behavioral problems and developmental retardation. About two-thirds of aniridia cases are familial, as the result of dominantly inherited mutations or deletions of the PAX6 gene (3). About $90 \%$ of the reported PAX6 mutations lead to termination of protein translation (4), which generates truncated unstable and biologically inactive proteins.

\section{Highlights on the role of PAX6 in eye development}

The Pax 6 gene is located at $11 \mathrm{p} 13$ chromosome and encodes a highly conserved transcriptional regulator with two DNA binding domains and a transcriptional transactivation domain. Pax6 is expressed in all structures of the developing eye and in regions of the forebrain, hindbrain, cerebellum, and spinal cord (5-7), the olfactory system (8), and endocrine pancreas (9). Based on a very complex transcriptional regulation, Pax 6 displays highly complex spatiotemporal and quantitative expression patterns in all of these various developmental contexts $(10,11)$.

PAX6 plays a role at multiple stages during eye development. In early stages, PAX6 expression in the surface ectoderm is essential for formation of the lens, while at later stages, expression in the lens vesicle is necessary for lens fiber differentiation and crystallin gene expression (reviewed in refs. 11, 12). Correct PAX6 dosage is critical for the development of eye and brain, and both overexpression and haploinsufficiency have phenotypic consequences. In humans, PAX6 haploinsufficiency causes aniridia (13), while in mice, $\operatorname{Pax} 6$ haploinsufficiency results in the small eye (Sey) phenotype (heterozygous $\mathrm{Pax}^{\mathrm{Sey+} /-}$ mice) (14). Homozygous mice (Pax6 $6^{\text {sey/sey }}$ mice) die in the perinatal stage and present with multiple severe brain abnormalities, no eyes, and no nasal structures $(6,14-16)$. Overexpression of the PAX6 gene also results in eye and brain malformations $(17,18)$, confirming that the appropriate level of expression is crucial for proper development. Inappropriate expression of Pax 6 is even able to induce ectopic eyes in flies and frogs (19, 20). Postnatally, strong expression of Pax6 is detected in neurons of various brain regions, including the olfactory bulb $(7,21)$. In both embryonic and adult neurogenesis, Pax6 is an important regulator of neural stem cell proliferation and differentiation into neurons $(12,22)$. Although knowledge of PAX6 functions in differentiated cell types remains very scarce, it is now clear that PAX6 is maintained in several cell types of the adult eye (lens epithelium, corneal limbus, pigmented ciliary body, iris) and is implicated in self-renewal and regeneration of ocular structures (reviewed in refs. 11, 23).

\section{Ataluren-containing formulation reverses congenital eye defects}

In this issue of the JCI, Gregory-Evans et al. (24) report exciting data on postnatal manipulation of Pax 6 dosage through a mutation-independent nonsense mutation suppression strategy in the $\mathrm{Pax} 6^{\mathrm{Sey+} /-}$ mouse eye. The Pax $6^{\mathrm{Sey+} /-}$ mouse is a relevant model of human aniridia, with a naturally occurring Gly194X stop codon mutation (UGA) in the Pax6 gene (14). For in vivo studies, systemic administration of the nonsense suppression drug 3-[5-(2-fluorophenyl)-1,2,4-oxadiazol-3-yl] benzoic acid (ataluren, also known as PTC124) (25) was used. Systemic administration of ataluren had limited benefits on the cornea and induced an incomplete functional rescue. To increase the drug concentration in the eye, a topical application of $1 \%$ aqueous ataluren suspension was used. Use of this topical formulation resulted in a partial histological rescue of the retina and lens defects; however, these beneficial effects were accompanied by marked ocular irritation in the Pax6 $6^{\text {Sey+/- }}$ mouse. In order to improve particle dispersion properties and increase suspension viscosity and ocular exposure, a unique ataluren-containing formulation was designed, referred to as START $(0.9 \%$ sodium chloride, $1 \%$ Tween $80,1 \%$ powdered ataluren, $1 \%$ carboxymethylcellulose). Importantly, START not only abolished the irritation but also reversed lens and retinal defects in the Pax $6^{\text {Sey+/- }}$ eyes. The effects were greater than those induced by ataluren alone, administered either topically or systemically, and the rescue of the corneal deficit achieved WT characteristics. The ELISA analysis of START-treated $\operatorname{Pax} 6^{\text {Sey+/- }}$ retinal and corneal epithelium protein lysates revealed that Pax6 protein levels increased to $90 \% \pm 5 \%$ of WT following treatment. Impressively, START therapy improved functional deficits of the retina, as measured both by electroretinography scotopic b-wave amplitudes and a behavioral optokinetic tracking test. In both cases, there was substantial benefit from START therapy compared with the other tested treatments. Furthermore, START promoted responses that were similar to those measured in WT animals. The effects of ataluren and the START formulation were compared with those of gentamicin, an aminoglycoside antibiotic known to suppress stop codons in vitro and in vivo (26). As expected, treatment with gentamicin resulted in remarkable normalization of the eye malformation defects. Because Sey mice that are homozygous for Pax6 mutations (Pax6 $6^{\text {Sey-1Nen }}$ mice) did not respond to START therapy, the authors suggested that this treatment specifically targets nonsense mutations.

\section{Ataluren for genetic disorders caused by nonsense mutations}

The original identification of ataluren arose from a cell-based luciferase assay of nonsense codon suppression and demonstration of selective ribosomal readthrough of PTCs (25). Initially, ataluren was determined to be effective in models of DMD (25). Ataluren treatment was shown to promote production of full-length and functionally active dystrophin in primary muscle cells from humans and mice expressing dystrophin nonsense alleles. Furthermore, ataluren administration rescued striated muscle function in a mouse model of DMD. The effect of ataluren in this study was found to be superior to gentamicin. Aminoglycoside antibiotics were previously shown to promote in vivo readthrough of nonsense mutations, resulting in expression of full-length proteins and/or correction of the protein function (26-28). Encouraging results were also obtained in models of CF, in which ataluren effectively suppressed the hCFTR-G542X nonsense mutation in vivo (29). Based on these proofof-concept studies, human clinical trials have been undertaken. The safety profile of single- and multiple-dose administration of ataluren was demonstrated in healthy adult volunteers (30). The oral administration of ataluren suppressed nonsense mutations and reduced the epithelial electrophysiological abnormalities caused by CFTR dysfunc- 
tion in patients with CF $(31,32)$. Because ataluren is not specific to a gene but to a type of mutation, it is believed that it could be an efficient treatment for many other disorders that are associated with nonsense mutations. Indeed, correction of nonsense bone morphogenetic protein receptor (BMPR2) and SMAD9 mutations by ataluren in lungand blood-derived cells from patients with pulmonary arterial hypertension has also been demonstrated (33).

\section{Doubts on the mechanism underlying the effect of ataluren}

Recently, the effectiveness of ataluren as a nonsense suppression agent has been questioned. In their initial characterization, Welch et al. described upregulation of luciferase reporter activity in response to ataluren, which they attributed to readthrough of the PTC (25). Other studies found that ataluren can increase luciferase reporter activity independently of PTC readthrough $(34,35)$. McElroy et al. (36) used a panel of PTC reporter assays, including transient transfection, stable cell lines, plate-based functional enzyme assays, and direct protein detection to compare activity of ataluren to the well-characterized readthrough activity of geneticin. In all of these assays, the activity of geneticin was documented, while ataluren had no measurable effects. Dual mechanisms of action for ataluren, a molecular target other than the luciferase reporter (35), or differences in the in vivo and in vitro activity of ataluren (36) have all been suggested as the source of disparity in these controversial findings. It should be considered also that multiple factors can affect the response to readthrough treatments, including identity of the PTC and its sequence context, the chemical composition of the readthrough drug, the level of PTC-bearing transcripts, and variability in nonsense-mediated mRNA efficiency (reviewed in ref. 37). In fact, while the underlying mechanism of ataluren action remains unclear, the efficacy of this drug has been confirmed both in animal models and clinical trials, particularly those focused on CF $(29,31)$. Further evidence of ataluren-mediated translational readthrough efficacy was reported in Usher syndrome type 1C. For this disease, ataluren treatment of cell culture, retinal explant, and in vivo models has been demonstrated to recover harmonin expression and restore harmonin scaffolding function (38). Cultured cells from patients with infantile neuronal ceroid lipofuscinosis, which is the result of nonsense mutations in palmitoyl protein thioesterase 1 (PPT1), exhibited a restoration of PPT1 enzyme activity following ataluren treatment that was virtually identical to the PPT1 enzyme activity restored by gentamicin treatment (39). Another word of caution should be expressed regarding the specificity of ataluren and its possible off-target undesirable effects.

\section{Exon skipping versus readthrough}

Theoretically, the readthrough approach is applicable to all nonsense mutations; however, an exon-skipping approach is specific to certain types of mutations, including deletion, nonsense, splice site, and duplication mutations (reviewed in ref. 40). Successful exon skipping requires specific oligos that are designed for each exon and use small synthetic modified RNA or DNA molecules (antisense oligonucleotides [AO]). The AOs must be able to recognize, bind, and mask the targeted exon from the splicing machinery and promote specific exon exclusion from the mature mRNA (41). The entire exon-skipping process leads to restoration of the genetic code and the protein function. Most nonsense mutations in the $D M D$ gene can be treated either with exon skipping or the readthrough strategy discussed above, with both strategies being tested in clinical trials. A first trial in patients with DMD reported that treatment with different doses of ataluren daily for 4 weeks was well tolerated; however, ataluren failed to meet statistical significance in its primary end point, which was improvement in the six-minute walking distance (6MWD) test within the 48-week duration of the phase IIb trial. It should be noted that ataluren slowed the loss of walking ability, as measured by the time to persistent $10 \%$ worsening in 6MWD (42). Similarly, drisapersen (also known as GSK2402968 and PRO051), an investigational AO currently in late-stage development for treatment of patients with DMD, did not provide significant functional improvement (as measured by the 6MWD test) compared with placebo in a phase III clinical study (43).

\section{Conclusion}

The mechanism(s) of action underlying the effects of ataluren and its functional efficacy remain to be fully determined. Despite controversial data from animal and human studies, readthrough mutation-targeted therapies, in principle, hold promise as a common therapeutic paradigm for a large number of genetic disorders that are caused by nonsense mutations. The study by Gregory-Evans et al. (24) demonstrates that the ataluren-containing START formulation promoted the synthesis of a full-length PAX6 protein and stably reversed congenital ocular malformations in postnatal mouse eye. These new findings offer additional experimental support for the beneficial effects of ataluren in genetic diseases caused by nonsense mutation and some basis for extending the therapeutic potential of ataluren toward congenital eye malformations.

Address correspondence to: José-Alain Sahel, Institut de la Vision, 17 rue Moreau, 75012, Paris, France. Phone: 33153462504. Fax: 33140021499. E-mail: jose-alain. sahel@upmc.fr.

1. Frischmeyer PA, Dietz HC. Nonsense-mediated mRNA decay in health and disease. Hum Mol Genet. 1999;8(10):1893-1900.

2. Mort M, Ivanov D, Cooper DN, Chuzhanova NA. A meta-analysis of nonsense mutations causing human genetic disease. Hum Mutat. 2008;29(8):1037-1047.

3. Hingorani M, Hanson I, van Heyningen V. Aniridia. Eur J Hum Genet. 2012;20(10):1011-107.

4. Prosser J, van Heyningen V. PAX6 mutations reviewed. Hum Mutat. 1998;11(2):93-108.

5. Walther C, Gruss P. Pax-6, a murine paired box gene, is expressed in the developing CNS. Development. 1991;113(4):1435-1449.

6. Warren N, Caric D, Pratt T, Clausen JA, Asavaritikrai P, Mason JO, Hill RE, Price DJ. The transcription factor, Pax6, is required for cell proliferation differentiation in the developing cerebral cortex. Cereb Cortex. 1999;9(6):627-635.

7. Stoykova A, Gruss P. Roles of Pax-genes in developing and adult brain as suggested by expression patterns. J Neurosci. 1994;14(3 pt 2):1395-1412.

8. Grindley JC, Davidson DR, Hill RE. The role of Pax-6 in eye nasal development. Development. 1995; 121(5):1433-1442.

9. Dohrmann C, Gruss P, Lemaire L. Pax genes and the differentiation of hormone-producing endocrine cells in the pancreas. Mech Dev. 2000;92(1):47-54.

10. Kleinjan DA, et al. Long-range downstream enhancers are essential for Pax6 expression. Dev Biol. 2006;299(2):563-581.

11. Shaham O, Menuchin Y, Farhy C, Ashery-Padan R. Pax6: a multi-level regulator of ocular development. Prog Retin Eye Res. 2012;31(5):351-376.

12. Osumi N, Shinohara H, Numayama-Tsuruta K, Maekawa M. Concise review: Pax6 transcription factor contributes to both embryonic adult neurogenesis as a multifunctional regulator. Stem Cells. 2008; 26(7):1663-1672.

13. Ton CC, et al. Positional cloning and characterization of a paired box- and homeobox-containing gene from the aniridia region. Cell. 1991;67(6):1059-1074.

14 . Hill RE, et al. Mouse small eye results from mutations in a paired-like homeobox-containing gene. Nature. 1991;354(6354):522-525.

15. Hogan BL, Horsburgh G, Cohen J, Hetherington CM, Fisher G, Lyon MF. Small eyes (Sey): a homozygous lethal mutation on chromosome 2 which affects the differentiation of both lens nasal placodes in the mouse. J Embryol Exp Morphol. 1986;97:95-110.

16. Stoykova A, Fritsch R, Walther C, Gruss P. Forebrain patterning defects in Small eye mutant mice. Development. 1996;122(11):3453-3465.

17. Schedl A, et al. Influence of PAX6 gene dosage 
on development: overexpression causes severe eye abnormalities. Cell. 1996;86(1):71-82.

18. Manuel M, Pratt T, Liu M, Jeffery G, Price DJ. Overexpression of Pax6 results in microphthalmia, retinal dysplasia defective retinal ganglion cell axon guidance. BMC Dev Biol. 2008;8:59.

19. Halder G, Callaerts P, Gehring WJ. Induction of ectopic eyes by targeted expression of the eyeless gene in Drosophila. Science. 1995;267(5205):1788-1792.

20. Chow RL, Altmann CR, Lang RA, Hemmati-Brivanlou A. Pax6 induces ectopic eyes in a vertebrate. Development. 1999;126(19):4213-4222.

21. Kawano H, et al. Pax- 6 is required for thalamocortical pathway formation in fetal rats. J Comp Neurol. 1999;408(2):147-160.

22. Xu S, et al. The proliferation and expansion of retinal stem cells require functional Pax6. Dev Biol. 2007; 304(2):713-721.

23. Karl MO, Reh TA. Regenerative medicine for retinal diseases: activating endogenous repair mechanisms. Trends Mol Med. 2010;16(4):193-202.

24. Gregory-Evans CY, Wang X, Wasan KM, Zhao J, Metcalfe AL, Gregory-Evans K. Postnatal manipulation of Pax 6 dosage reverses congenital tissue malformation defects. J Clin Invest. 2014;124(1):111-116.

25. Welch EM, et al. PTC124 targets genetic disorders caused by nonsense mutations. Nature. 2007 447(7140):87-91.

26. Barton-Davis ER, Cordier L, Shoturma DI, Leland SE, Sweeney HL. Aminoglycoside antibiotics restore dystrophin function to skeletal muscles of $\mathrm{mdx}$ mice. JClin Invest. 1999;104(4):375-381.

27. Howard M, Frizzell RA, Bedwell DM. Aminoglycoside antibiotics restore CFTR function by overcoming premature stop mutations. Nat Med. 1996; 2(4):467-469.

28. Wagner KR, et al. Gentamicin treatment of Duchenne and Becker muscular dystrophy due to non- sense mutations. Ann Neurol. 2001;49(6):706-711.

29. Du M, Liu X, Welch EM, Hirawat S, Peltz SW, Bedwell DM. PTC124 is an orally bioavailable compound that promotes suppression of the human CFTR-G542X nonsense allele in a CF mouse model Proc Natl Acad Sci U S A. 2008;105(6):2064-2069.

30. Hirawat $S$, et al. Safety, tolerability, and pharmacokinetics of PTC124, a nonaminoglycoside nonsense mutation suppressor, following single- multipledose administration to healthy male female adult volunteers. J Clin Pharmacol. 2007;47(4):430-444

31. Kerem E, et al. Effectiveness of PTC124 treatment of cystic fibrosis caused by nonsense mutations: a prospective phase II trial. Lancet. 2008 372(9640):719-727.

32. Sermet-Gaudelus I, et al. Ataluren (PTC124) induces cystic fibrosis transmembrane conductance regulator protein expression activity in children with nonsense mutation cystic fibrosis. Am J Respir Crit Care Med. 2010;182(10):1262-1272.

33. Drake KM, Dunmore BJ, McNelly LN, Morrell NW, Aldred MA. Correction of nonsense BMPR2 and SMAD9 mutations by ataluren in pulmonary arterial hypertension. Am J Respir Cell Mol Biol. 2013; 49(3):403-409.

34. Auld DS, Thorne N, Maguire WF, Inglese J. Mechanism of PTC124 activity in cell-based luciferase assays of nonsense codon suppression. Proc Natl Acad Sci U S A. 2009;106(9):3585-3590.

35. Auld DS, et al. Molecular basis for the high-affinity binding and stabilization of firefly luciferase by PTC124. Proc Natl Acad Sci U S A. 2010; 107(11):4878-4883

36. McElroy SP, et al. A lack of premature termination codon read-through efficacy of PTC124 (Ataluren) in a diverse array of reporter assays. PLoS Biol. 2013;11(6):e1001593.

37. Linde L, Kerem B. Introducing sense into nonsense in treatments of human genetic diseases. Trends Genet. 2008;24(11):552-563.

38. Goldmann T, Overlack N, Wolfrum U, Nagel-Wolfrum K. PTC124-mediated translational readthrough of a nonsense mutation causing Usher syndrome type 1C. Hum Gene Ther. 2011;22(5):537-547.

39. Sarkar C, Zhang Z, Mukherjee AB. Stop codon readthrough with PTC124 induces palmitoyl-protein thioesterase-1 activity, reduces thioester load suppresses apoptosis in cultured cells from INCL patients. Mol Genet Metab. 2011;104(3):338-345.

40. Yokota T, Duddy W, Echigoya Y, Kolski H. Exon skipping for nonsense mutations in Duchenne muscular dystrophy: too many mutations, too few patients? Expert Opin Biol Ther. 2012;12(9):1141-1152.

41. Pichavant $C$, et al. Current status of pharmaceutical and genetic therapeutic approaches to treat DMD. Mol Ther. 2011;19(5):830-840.

42. PTC Therapeutics. Pivotal data presented at the world muscle society congress suggest ataluren slows the loss of walking ability in patients with nonsense mutation. Duchenne/Becker Muscular Dystrophy. PR Newswire Web site. http://www.prnewswire.com/news-releases/ pivotal-data-presented-at-the-world-musclesociety-congress-suggest-ataluren-slows-the-lossof-walking-ability-in-patients-with-nonsensemutation-duchennebecker-muscular-dystrophy105019789.html. Updated October 15, 2010. Accessed November 18, 2013.

43. GlaxoSmithKline. GSK Prosensa announce primary endpoint not met in Phase III study of drisapersen in patients with Duchenne Muscular Dystrophy. GSK Web site. http://www.gsk.com/media/ press-releases/2013/gsk-and-prosensa-announceprimary-endpoint-not-met-in-phase-iii-.html. Updated September 20, 2013. Accessed November 18,2013 